\title{
Forma Atípica de Tireoidite Supurativa Aguda em Paciente Pediátrico: Relato de Caso
}

\begin{abstract}
RESUMO
A tireoidite aguda é uma doença rara. Na infância associa-se principalmente a anormalidades congênitas com acometimento do lobo esquerdo. Na ausência de tireoidopatia preexistente, a função tireoidiana geralmente está normal. Relatamos um caso de uma menina, 6 anos de idade, com tumoração associada a sinais flogísticos na região cervical anterior há 15 dias acompanhada de leucocitose com desvio à esquerda, VSH e TSH elevados. Não havia história prévia de tireoidopatia. A ultra-sonografia da região cervical foi sugestiva de abscesso tireoidiano. Realizou-se antibioticoterapia e drenagem cirúrgica com boa resposta, porém posteriormente a paciente evoluiu com hipotireoidismo clínico. O esofagograma não detectou fístula do seio piriforme e a ultra-sonografia controle revelou heterogeneidade do parênquima, levantando-se a possibilidade de tireoidite de Hashimoto. A importância do caso deve-se à sua apresentação incomum: ausência de anormalidades congênitas, acometimento difuso da tireóide, alteração da função tireoidiana e provável tireoidite de Hashimoto como fator predisponente da tireoidite aguda. Arq Bras Endocrinol Metab 2008; 52/4:701-706)
\end{abstract}

Descritores: Tireoidite aguda; Abscesso tireoidiano; Tireoidite de Hashimoto; Criança; Hipotireoidismo; Ultra-sonografia.

\section{ABSTRACT}

Atypical Form of Acute Suppurative Thyroiditis in Pediatric Patient: Case Report. Acute thyroiditis is an unusual disease. In pediatric patients it is usually associated with congenital abnormalities towards the unilateral envelopment of the left thyroid lobe. In general, in the absence of preexisting thyroid disease, the thyroid function is most often normal. It is here described a case of a sixyear-old girl who had presented a mass with flogistic signs in the anterior neck region for fifteen days, together with leukocytosis and increased levels of VSH and TSH. There was no evidence of previous thyroid disease and the cervical ultrasonography suggested thyroid abscess. Intravenous antibiotic and surgical dranaige were done with a good response, however, the patient developed clinical hypothyroidism aftherwards. Barium swallow did not demonstrate pyriform sinus fistula and the follow-up ultrasonography revealed heterogeneous internal texture suggesting Hashimoto's Thyroiditis. The relevance of this case is due to its unusual findings: the absence of congenital abnormalities, bilateral envelopment of thyroid lobes, hypothyroidism and a probable Hashimoto's Thyroiditis as a predisposing factor for acute thyroiditis. Arq Bras Endocrinol Metab 2008; 52/4:701-706)

Keywords: Acute Thyroiditis; Abscess thyroid; Child; Hypothyroidism; Hashimoto's thyroiditis; Ultrasonography.

\section{apresentação de caso}

\author{
MARIA RosineIDE dos S. TORRES \\ CARLA C. M. MEDEIROS \\ Sebastião Horácio Nobrega Neto \\ LINCOLN S. SOUZA \\ MÔNICA L. C. RODRIGUes \\ Maria Nelice M. Silva \\ LYZANDRA DE H. C. S. MAIOR \\ Márcia C. Avelino \\ Germana T. F. MourÃo
}

Departamento de Endocrinologia (MRST); Serviço de Pediatria e Endocrinologia (CCMM); Serviço de Ultrasonografia (SHNN); Serviço de Cirurgia de Cabeça e Pescoço (LSS), Pediatria (MLCR, MNMS); da Faculdade de Ciências Médicas da Universidade Federal de Campina Grande (UFCG) (LHCSM, MCA, GTFM), $\mathrm{PB}$, Brasil.

Recebido em 24/04/2007 Aceito em 22/02/2008 


\section{INTRODUÇÃO}

A TIREOIdite AGUdA (TA) É uma doença rara, com não mais que um a dois casos observados por ano em hospital de atendimento terciário. Esta baixa incidência deve-se a mecanismos de proteção da tireóide, como amplo suprimento sangüíneo e linfático, proteção anatômica exercida por sua cápsula e alta concentração de iodo no parênquima tireoidiano (1).

Uma gama de agentes etiológicos já foram descritos (1-4), todavia, os principais patógenos envolvidos são estreptococos e estafilococos (5). A infecção usualmente se expande para a tireóide de maneira direta por meio de estruturas adjacentes, ou a distância, por meio do sistema sangüíneo ou linfático. Em pacientes pediátricos, as principais fontes de infecções são anomalias congênitas, como persistência do ducto tireoglosso e fístula do seio piriforme, levando principalmente ao acometimento tireoidiano unilateral esquerdo $(6,7)$ e, em alguns casos, recorrência do quadro (5). Já no adulto, a disseminação hematogênica e linfática de infecção oriunda de vias aéreas superiores ou a distância para uma tireóide com alteração preexistente são as causas mais freqüentemente postuladas (8).

As manifestações clínicas mais comuns são dor na região cervical anterior, aumento de volume da tireóide, podendo estar acompanhado de febre e sinais de compressão local. O lobo esquerdo é o mais freqüentemente envolvido principalmente na presença de fístula do seio piriforme, porém em algumas ocasiões, como na presença de doença tireoidiana preexistente, ambos os lobos podem estar acometidos (9).

$\mathrm{Na}$ maioria dos casos ocorre a integridade da função tireoidiana $(1,6,7)$ com anticorpos antitireoidianos, antiperoxidade (anti-TPO) e antitiroglobulina (anti$\mathrm{TG}$ ), geralmente indectáveis (10), porém quando o comprometimento é difuso, como nos casos de infecções fúngicas ou por microbactérias, e nos portadores de doença tireoidiana prévia, pode ocorrer tireotoxicose ou hipotireoidismo $(1,11)$.

A punção aspirativa por agulha fina (PAAF) e a cultura do aspirado são procedimentos diagnósticos fundamentais quando se suspeita desta doença $(1,12)$.

A terapêutica apropriada dependerá da etiologia microbiológica de cada caso. O tratamento cirúrgico pode variar desde a drenagem ou ressecção cirúrgica por meio de pequena incisão para a colocação do dreno até raros casos de tireoidectomia parcial ou total. Fístu- las do seio piriforme devem ser removidas para prevenir recorrências da doença $(1,5)$.

As infecções tireoidianas são potencialmente letais, dependendo o seu prognóstico de um pronto reconhecimento e um tratamento adequado (12).

O objetivo deste trabalho é descrever o caso de uma criança com diagnóstico de tireoidite aguda, mesmo na ausência de anormalidades congênitas, que apresentou um acometimento difuso da glândula tireóide e evoluiu com hipotireoidismo, diferindo da maioria dos casos relatados na literatura.

\section{RELATO DE CASO}

Paciente, J.M.B.B., sexo feminino, 6 anos, natural de Esperança, PB, foi internada no hospital universitário com história de otalgia bilateral acompanhada de disfagia, odinofagia e febre há 15 dias associada à tumoração volumosa e dolorosa na região anterior do pescoço, na topografia da tireóide. Apresentava ainda anorexia, perda ponderal e vinha em uso de cefalexina, por uma otite previamente diagnosticada, há mais ou menos oito dias, sem melhora do quadro. A história de doença tireoidiana preexistente, trauma, irradiação cervical e antecedente familiar de tireoidopatia foram negativos.

Quando admitida, a paciente encontrava-se com estado geral comprometido, eupneica, afebril, apresentando aumento de volume da região cervical anterior com hiperemia, calor local e dor intensa à palpação da tireóide (Figura 1). Não existia alteração à inspeção da orofaringe

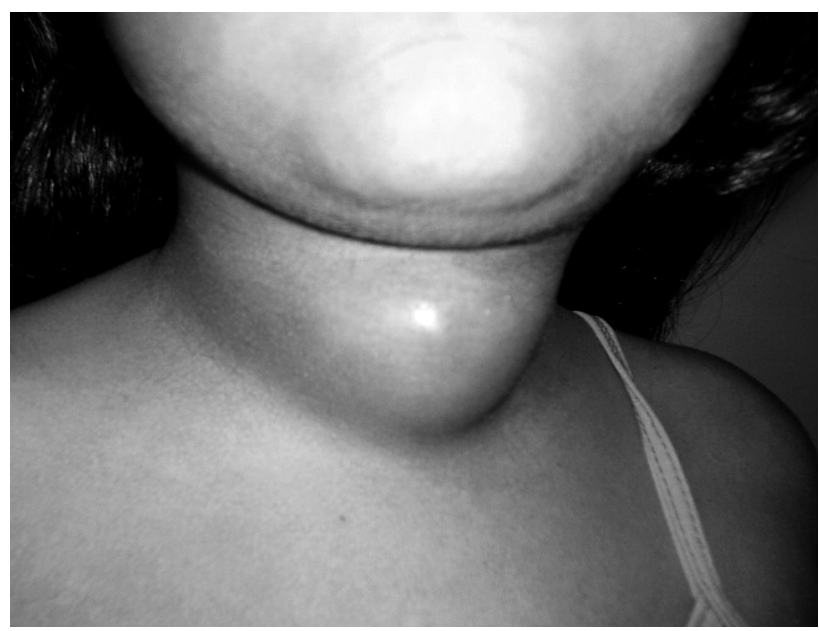

Figura 1. Região cervical anterior apresentando tumoração com sinais flogísticos em razão do acometimento inflamatório difuso da glândula tireoidiana. 
nem à otoscopia. Os exames laboratoriais mostraram: hemograma com leucocitose, 12.100 leucócitos $/ \mathrm{mm}^{3}$ (valor de referência: 5.000-10.000 leucócitos $/ \mathrm{mm}^{3}$ ) e desvio à esquerda, $\mathrm{VSH}=57 \mathrm{~mm} / \mathrm{h}$ (valor de referência: $0-13 \mathrm{~mm} / \mathrm{h}$ ), TSH = 21,8 $\mu \mathrm{UI} / \mathrm{ml}$ (valor de referência: $0,3-4,2 \mu \mathrm{UI} / \mathrm{ml}$ ) e T4 $4_{\text {livre }}=0,97 \mathrm{ng} / \mathrm{dl}$ (valor de referência: $0,8-1,9 \mathrm{ng} / \mathrm{dl}$ ) caracterizando um hipotireoidismo subclínico. As mensurações séricas do TSH e T4 ${ }_{\text {livre }}$ foram realizadas pelo método de quimioluminescência (kit DPC-IMMULITE).

A ultra-sonografia (USG) da região cervical foi realizada com aparelho da marca Toshiba Nemiol7 utilizando-se transdutor linear multifreqüencial de 6 a $12 \mathrm{MHz}$, que detectou aumento difuso da glândula tireóide, contornos irregulares e heterogeneidade difusa do parênquima, com áreas hipoecóicas mal definidas e dispersas. Foi ainda identificada uma formação expansiva hipoecóica de contornos parcialmente definidos, medindo cerca de $2,4 \times 2,1 \mathrm{~cm}$ nos maiores diâmetros, com focos puntiformes hiperecogênicos no seu interior ocupando a quase totalidade do lobo direito (lesão cística com conteúdo de líquido espesso) associada a acentuado edema de partes moles adjacentes. Estes achados são sugestivos de processo inflamatório da glândula tireóide com área de liquefação no lobo direito, possivelmente relacionado a abscesso (Figura 2). O lobo direito mediu 4,8 $\times 2,6 \times 2,4 \mathrm{~cm}$ de diâmetro e o lobo esquerdo $4,3 \times 2,4 \times 2,4 \mathrm{~cm}$ de diâmetro com volume tireoidiano igual a $30,0 \mathrm{~cm}^{3}$ (valor normal para estatura $4,9 \mathrm{~cm}^{3} \pm 1,1 \mathrm{~cm}^{3}$ ).

Diante deste resultado e da gravidade do quadro, iniciou-se a terapêutica com clindamicina e ceftriaxona, associados à levotiroxina pelo hipotireoidismo subclínico. No segundo dia de antibioticoterapia, a paciente evoluiu com aumento da dor e do volume da tumoração na região cervical, substituindo-se então a clindamicina pela oxacilina. No quarto dia de internação, exteriorizou-se um abscesso com área de flutuação, tendo sido realizada drenagem cirúrgica e retirada de grande quantidade de secreção purulenta. A cultura do conteúdo coletado apresentou-se negativa, provavelmente pelo uso prévio de antibiótico. A paciente recebeu alta hospitalar fazendo uso, em regime domiciliar, de levotiroxina $50 \mathrm{mcg} /$ dia e cefalexina para término do esquema da antibioticoterapia.

Após um mês, a paciente retorna com bom estado geral e ausência de sinais flogísticos na região afetada (Figura 3). A USG de controle, realizada pelo mesmo operador em aparelho com as mesmas especificações anteriores, revelou área focal hipoecogênica no lobo direito, com lobo esquerdo e istmo sem alterações ultra-sonográficas e com volume tireoidiano dentro da normalidade. A pesquisa de fístula do seio piriforme, por meio do esofagograma, foi negativa. $\mathrm{O}$ controle da função tireoidiana ainda mostrou descompensação com TSH $=7,68 \mu \mathrm{UI} / \mathrm{ml}$ (valor de referência: $0,3-4,2 \mu \mathrm{UI} / \mathrm{ml}$ ) e T4 $4_{\text {live }}=0,29 \mathrm{ng} / \mathrm{dl}$ (valor de referência: 0,8-1,9 ng/dl), sendo necessário o aumento da dosagem de levotiroxina para $75 \mathrm{mcg} / \mathrm{dia}$. A mensuração do anti-TPO realizada pelo método de quimioluminescência (kit DPC-Immulite) foi inferior a $10 \mathrm{UI} / \mathrm{ml}$ (valor de referência: menor que $15 \mathrm{UI} / \mathrm{ml})$.
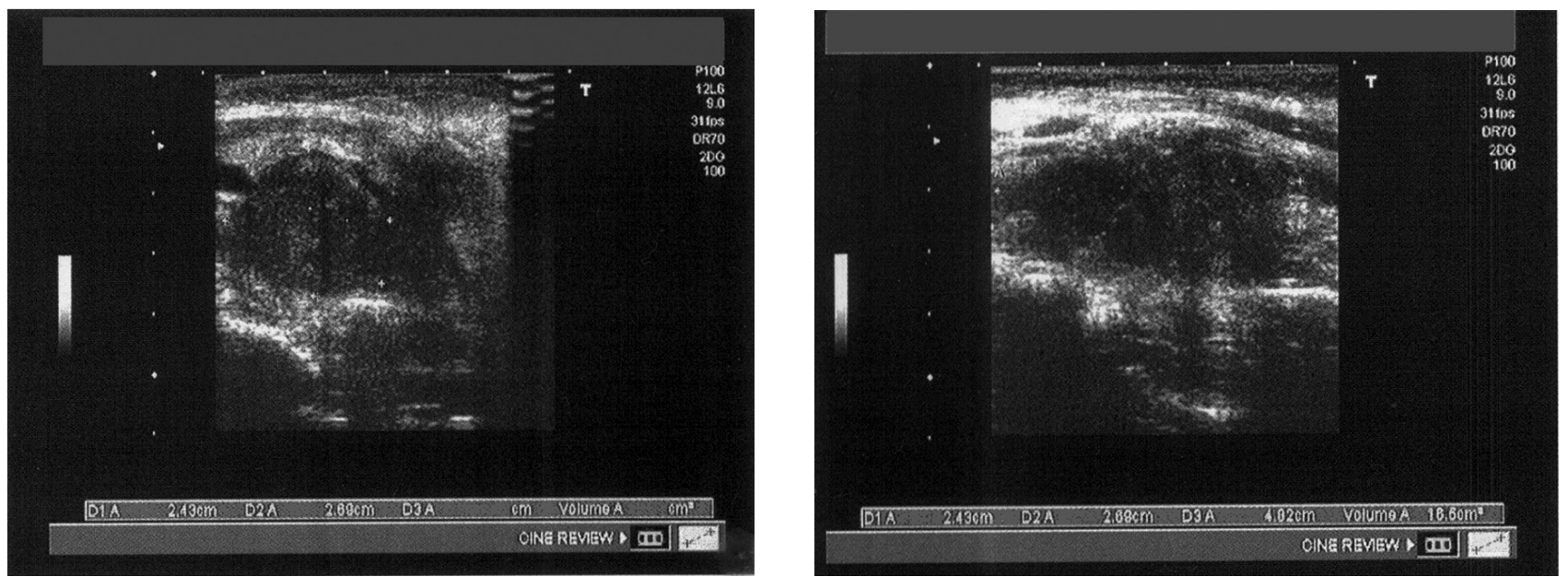

Figura 2. Ultra-sonografia tireoidiana mostrando heterogeneidade difusa do parênquima, com áreas hipoecóicas mal definidas e formação expansiva hipoecóica no lobo direito sugerindo processo inflamatório da glândula com área de liquefação possivelmente relacionada a abscesso tireoidiano. 


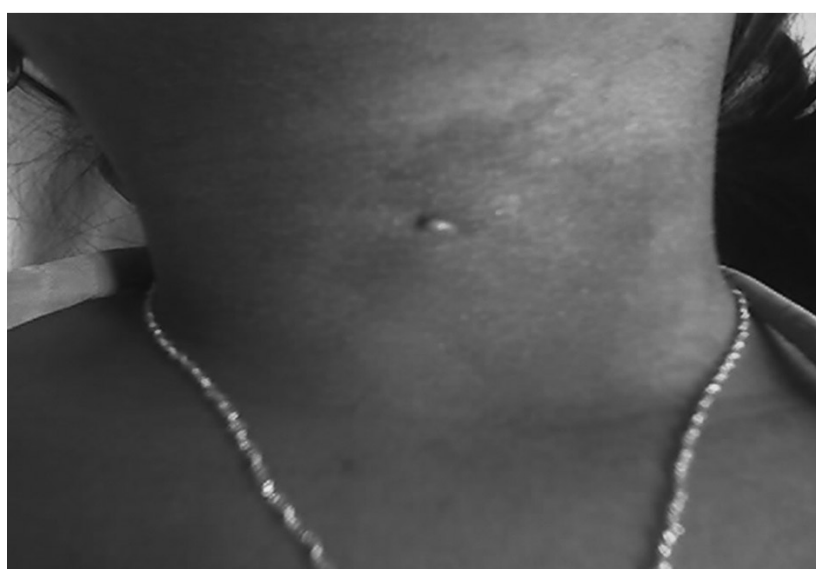

Figura 3. Região cervical um mês após a resolução do quadro de tireoidite aguda.

Após 1 ano e 6 meses do diagnóstico de TA, a paciente continua em uso de $75 \mathrm{mcg} / \mathrm{dl}$ de levotiroxina com TSH e $\mathrm{T}_{\text {livre }}$ dentro da normalidade e anticorpos antitireoidianos negativos, anti-TPO com valor inferior a $10 \mathrm{UI} / \mathrm{ml}$ (valor de referência: até $35 \mathrm{UI} / \mathrm{ml}$ ) e anti-TG igual a 1,6 UI/ml (valor de referência: menor que 40), ambas as mensurações realizadas pelo método de quimioluminescência (kit DPC-Immulite). A USG de seguimento, realizada com os mesmos parâmetros anteriores, mostrou lobos tireoidianos de contornos regulares com textura heterogênea, identificando-se uma área de conformação nodular, hipoecóica com contornos parcialmente definidos, localizada no terço médio do lobo direito, medindo cerca de $0,9 \mathrm{~cm}$ em seu maior diâmetro e com vascularização apenas na periferia, ao estudo doppler color. O lobo direito mediu $3,0 \times 1,2 \times 0,8 \mathrm{~cm}$ de diâmetros e lobo esquerdo $2,6 \times 1,0 \times 0,8 \mathrm{~cm}$ de diâmetros com volume tireoidiano igual a $3,2 \mathrm{~cm}^{3}$ (valor normal para estatura $4,9 \mathrm{~cm}^{3} \pm 1,1 \mathrm{~cm}^{3}$ ) ( Figura 4).

\section{DISCUSSÃo}

Recentemente, têm-se observado aumento dos casos de tireoidite supurativa provavelmente em virtude do aumento do número de imunocomprometidos (1). A TA é mais comum em crianças do que em adultos, nos portadores de doença tireoidiana prévia (neoplasia tireoidiana, tireoidite de Hashimoto (TH) e bócio multinodular) ou naqueles com anomalias congênitas, como persistência do ducto tireoglosso ou presença de fístula do seio piriforme; sendo esta última condição mais comum em crianças $(5,11,13)$. Aproximadamente

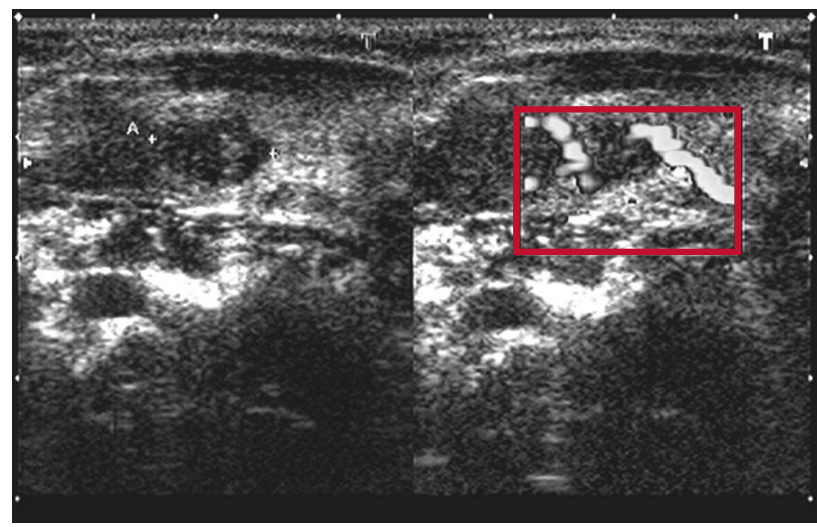

Figura 4. Ultra-sonografia de seguimento, 1 ano e 6 meses após o quadro de tireoidite, mostrando textura heterogênea da tireóide e área de conformação nodular, hipoecóica com contornos parcialmente definidos localizada no terço médio do lobo direito, caracterizando pseudonódulo, achado comum na tireoidite de Hashimoto.

a metade dos pacientes com TA tem doença tireoidiana preexistente (11).

Entre as doenças tireoidianas na infância, a TA tem prevalência de $1,6 \%$, sendo mais rara que a TH $(98,2 \%)$, porém mais comum do que a tireoidite subaguda (14), ao contrário do que ocorre no adulto em que a tireoidite subaguda é mais freqüente do que a TA (1).

$\mathrm{Na}$ criança, o diagnóstico diferencial com abscesso cervical é de suma importância, uma vez que este e a TA são mais freqüentes nesta faixa etária, e às vezes manifestam-se de maneira semelhante, podendo ambos sucederem uma infecção de vias áreas superiores. Abscesso nos espaços retrofaríngeo, parafaríngeo, peritonsilar e submandibular podem apresentar-se com febre, edema e dor na região cervical, semelhante à TA, entretanto o diagnóstico diferencial dá-se por método de imagem (USG e/ou cintilografia) que mostra a tireóide normal. $\mathrm{O}$ abscesso retrofaríngeo pode ser diagnosticado apenas pela tomografia computadorizada (1).

$\mathrm{Na}$ população pediátrica, as infecções de vias aéreas superiores e as tonsilites são as causas mais comuns de abscesso cervical, sendo os espaços retrofaríngeo e parafaríngeo os mais acometidos, principalmente em crianças acima de 1 ano (15).

Os agentes etiológicos mais comuns dos abscessos cervicais são os estreptococos e os estafilococos, assim como na TA, porém a média de idade do acometimento é um pouco inferior $(4,1$ anos) à da TA $(6$ anos $)$, e $75 \%$ dos casos ocorrem antes do 5 anos de idade $(6,7,15,16)$. Isso provavelmente é por causa da atrofia 
dos nódulos linfáticos após o terceiro e quarto ano de idade, uma vez que a formação do abscesso cervical dáse pela propagação de infecções da nasofaringe, dos seios paranasais e do ouvido médio para o espaço profundo do pescoço, diretamente por continuidade, ou por drenagem linfática para os nódulos linfáticos deste espaço (16).

Sepse, disseminação para o mediastino, recorrência, compressão de estruturas adjacentes e abscesso cervical são complicações da TA (12). Por outro lado, o abscesso cervical pode complicar-se com a TA (2).

No caso relatado, os achados que fundamentaram a suspeita clínica de TA foram história anterior de infecção de vias áreas superiores (otite), presente em 66\% (2) dos casos de TA; quadro clínico típico de dor e tumoração com características inflamatórias, sugestivos de formação de abscesso na região cervical anterior; leucocitose com desvio à esquerda e VSH elevado $(1,2,13)$. A USG mostrou-se de extrema importância na diferenciação entre abscesso cervical e tireoidiano, uma vez que identificou área de liquefação no interior do parênquima glandular, possivelmente pós-necrótica e não em partes moles adjacentes, configurando forte indício de abscesso tireoidiano.

O que chama a atenção neste caso é que o quadro, apesar de ocorrer em idade semelhante à média de idade citada na literatura, apresentou características clínicas incomuns. Na criança, a TA geralmente está associada a anomalias congênitas com acometimento do lobo esquerdo da tireóide e função tireoidiana normal $(1,5)$.

Onishi e cols.(7), no estudo clínico sobre TA em 15 crianças entre 3 e 14 anos com média de 6 anos, citam que em $11(73,3 \%)$ casos o lobo esquerdo foi afetado e em quatro $(26,6 \%)$, o lobo direito. Nenhum dos pacientes apresentou acometimento bilateral. Dos nove pacientes examinados, todos tinham função tireoidiana normal e quatro (44\%) possuíam fístula do seio piriforme. Do mesmo modo, Chi e cols. (6), em estudo com o mesmo número de crianças portadoras de TA e com a mesma média de idade, verificaram que $13(86,6 \%)$ tiveram o lobo esquerdo afetado e duas, o lobo direito $(13,3 \%)$. A função tireoidiana encontravase normal em todos os casos, e cinco $(33,3 \%)$ dos 15 pacientes investigados tinham fístula do seio piriforme no lado esquerdo. Nesta paciente, a pesquisa de fístula do seio piriforme foi negativa e houve acometimento difuso da tireóide associado a hipotireoidismo inicialmente subclínico, mas que evoluiu para hipotireoidismo clínico, o qual persiste até o momento.
O desenvolvimento inicial de hipotireoidismo subclínico é extremamente incomum, uma vez que, na fase aguda, o que pode ocorrer, e ainda assim excepcionalmente, são sintomas de tireotoxicose, sobretudo quando a tireoidite é difusa e há destruição intensa da glândula com extravasamento de hormônios para a circulação (1). Após controle do quadro infeccioso, a paciente evoluiu com hipotireoidismo clínico, achado incomum, já que a maioria dos casos de TA com alteração da função tireoidiana evolui para eutireoidismo em um a três meses (13). A persistência do hipotireoidismo pode ocorrer em algumas situações quando o acometimento da glândula é difuso, como nas tireoidites de etiologia fúngica, tuberculosa ou na presença de tireoidopatia prévia $(1,11)$ quando há destruição intensa da tireóide (1).

O acometimento difuso da tireóide com evolução na metade dos casos para hipotireoidismo pode ocorrer em portadores de doença tireoidiana preexistente. Neste caso, entretanto, não havia diagnóstico anterior de tireoidopatia, porém a USG de controle mostrou heterogeneidade do parênquima tireoidiano, levando ao provável diagnóstico de TH (17). A TH é a doença tireoidiana mais comum na infância, na qual os anticorpos antitireoidianos, anti-TPO e anti-TG são detectáveis em 97\% dos casos, com predomínio do anti-TPO.

A paciente, por sua vez, apresentou os anticorpos antitireoidianos negativos, porém isso não afasta o diagnóstico de $\mathrm{TH}$, uma vez que em crianças os títulos de anticorpos tendem a ser baixos e ocasionalmente negativos (17). Além disso, as alterações ultra-sonográficas podem preceder o aparecimento dos anticorpos (18).

Apesar de detectado ao USG uma área nodular hipoecóica com contornos parcialmente definidos, não foi realizada a PAAF, por se tratar de pseudonódulo, achado comum na TH (1).

Com base nestes dados, conclui-se que a paciente provavelmente seria portadora de tireoidopatia prévia (TH ou tireoidite linfocítica crônica) não diagnosticada, que atuou como fator predisponente para o desenvolvimento da TA. Por outro lado, o acometimento difuso da tireóide pode ter sido tão significativo que levou ao hipotireoidismo clínico que persiste até o momento.

Em relação ao tratamento, a paciente em questão teve boa resposta clínica à antibioticoterapia e à drenagem cirúrgica, condutas recomendadas independentemente do fator predisponente para a tireoidite aguda $(2,5)$. 
Portanto, o caso apresentado reveste-se de importância pelo fato de discutir uma doença que, além de rara, apresenta características peculiares e cujo prognóstico é extremamente dependente de uma ação clínicoterapêutica eficaz e imediata, a fim de evitar-se a perda da integridade da tireóide e garantir o sucesso clínico de maneira global.

\section{REFERÊNCIAS}

1. Lazarus J. Acute e subacute thyroiditis [acesso em 2007 Oct]. Disponível em: htpp://www.thyroidmanager.org/Chapter19/index.html.

2. Taylor WE Jr, Myer CM, Hays LL, Cotton RC. Acute suppurative thyroiditis in children. Laryngoscope. 1982;(92):1269-73.

3. Guerrero VG, Paulino A, Gonzáles FS, Sempere MM, Asensio VG, Villena JC. Abscesso laterocervical secundario a uma tiroiditis aguda supurada por gérmenes anaerobios. ORL-DIPS 2002; 29(3):135-7.

4. Park YH, Baik JH, Yoo J. Acute thyroiditis of actinomycosis. Thyroid. 2005;15(12):1395-6.

5. Sai Prasad TRS, Chong CL, Mani A, Chui CH, Tan CE, Tee WS, et al. Acute suppurative thyroiditis in children secondary to pyriform sinus fistula. Pediatr Surg Int. 2007;23(8):779-83.

6. Chi H, Lee YJ, Chiu NC, Huang FY, Huang CY, Lee KS, et al. Acute suppurative thyroiditis in children. Pediatr Infect Dis J. 2002; 21(5):384-7

7. Onishi H, Wataki K, Sasaki N, Niimi H. Clinical study of 15 children with acute suppurative thyroiditis. Nippon Naibunpi Gakkai Zasshi. 1994;20:70(5):529-35 (Abstract).

8. Sicilia V, Mezitis S. A case of acute suppurative thyroiditis complicated by thyrotoxicosis. J Endocrinol Invest. 2006;29(11):997-1000.

9. Dunham B, Nicol TL, Ishii M, Basaria S. Suppurative thyroiditis. Lancet. 2006;368(9548):1742.
10. Jr Foley T. Disorders of the thyroid in children. In: Sperling MA. Pediatric endocrinology. WB Saunders; 1996.

11. Bindra A, Braunstein GD. Thyroiditis. Am Fam Physician. 2006;73:1769-76.

12. Bertelii AAT, Andrade SJC, Kavabata NK, Menezes MB, Gonçalves AJ. Abscesso de tireóide tratado por punção: relato de caso e revisão de literatura. Rev Bras Cir Cabeça e Pescoço. 2005;34:23-5.

13. Montenegro RM, Montenegro Jr. RM, Vilar L. Tireoidite aguda. In: Vilar L. editor. Endocrinologia clínica. $3^{\text {a }}$ ed. Guanabara Koogan:, Rio de Janeiro 2006.

14. Wasniewska M, Vigone MC, Cappa M, Aversa T, Rubino M, De Luca F. Study group for thyroid diseases of italian sociaty for pediatris endocrinology. J Endocrinol Invest. 2007;30(4):346-7.

15. Coticchia JM, Getnick GS, Yun RD, Arnold JE. Age-site, and time-specific differences in pediatric deep neck abscesses. Arch Otolaryngol Head Neck Surg. 2004;130(2):201-7.

16. Craig FWM, Schunk JE. Retropharyngeal abscess in children: clinical presentation, utility of imaging and current management. Pediatrics. 2003;111:1394-8. [acesso em 2007 Sept. Disponível em: http://pediatrics.aapublications.org/cgi/111/6/1394.

17. Akamizu T, Amino N, De Groot LJ. Hashimoto's thyroiditis [acesso em 2007 Oct]. Disponível em: htpp://thyroidmanager. org/Chapter8/8-frame.htm.

18. Kabelitz M, Liesenkötter B, Stäch B, Willgerodt $H$, Stablein Singendonk $\mathrm{W}$, et al. The prevalence of anti-thyroid peroxidase antibodies and autoimmune thyroiditis in children and adolescents in an iodine replete area. Eur $\mathrm{J}$ Endocrinol. 2003;148:301-7.

\section{Endereço para correspondência:}

Carla Campos Muniz Medeiros

Rua Agamenon Magalhães n 230, apto. 1302 - Alto Branco 58102-589 Campina Grande, PB

E-mail: carlamunizmedeiros@hotmail.com 